\title{
Lapatinib in combination with capecitabine in the management of ErbB2-positive (HER2-positive) advanced breast cancer
}

\author{
Bella Kaufman' \\ Steven Stein ${ }^{2}$ \\ Michelle A Casey ${ }^{2}$ \\ Beth $O$ Newstat ${ }^{2}$ \\ 'Sackler Faculty of Medicine, \\ Tel Aviv University, Tel Aviv, Israel; \\ ${ }^{2}$ GlaxoSmithKline, Collegeville, PA, \\ USA
}

\begin{abstract}
Lapatinib is an oral, reversible, dual inhibitor of epidermal growth factor receptor ErbB1 (EGFR) and human epidermal growth factor receptor type 2 ErbB2 (HER2). Results of a phase III study comparing lapatinib plus capecitabine with capecitabine alone in women with ErbB2-overexpressing advanced breast cancer previously treated with an anthracycline, a taxane, and trastuzumab were reported early based on superiority of the combination in prolonging time to tumor progression (TTP). An updated analysis in 399 women supports the earlier findings. In this updated analysis, TTP (hazard ratio [HR] 0.57) favored lapatinib plus capecitabine. Survival trended in favor of the combination. The incidence of cardiac events was numerically higher in the combination arm ( 5 cases in the combination arm, 2 cases in the monotherapy arm).
\end{abstract}

Keywords: lapatinib, capecitabine, breast cancer, HER2

\section{Introduction}

Despite substantial advances in the treatment of breast cancer, it remains the most common cause of death in women 45-55 years old (American Cancer Society 2007). While only $10 \%$ of cases are advanced at the time of diagnosis, after primary treatment with curative intent, $10 \%-20 \%$ of patients will have local recurrence within 9 years, and $10 \%-25 \%$ will have locally extensive or metastatic disease (Heimann and Hellman 2000; ESMO Guidelines Working Group 2007).

Treatment of advanced disease is guided in large part by hormone-receptor status, ErbB2 (HER2) status, response to prior treatment, and co-existing conditions (eg, heart disease) that may or may not be related to prior therapy (eg, anthracyclines and trastuzumab).

Approximately $20 \%-25 \%$ of breast cancers overexpress human epidermal receptor type 2 (ErbB2 or HER2), which is associated with a more aggressive cancer and predicts poor clinical outcome (Slamon et al 1987). Currently, women with ErbB2-positive disease are treated with trastuzumab with or without chemotherapy (National Comprehensive Cancer Network 2007), but the optimal duration of treatment remains to be determined. For example, many patients who progress during or after trastuzumab-based therapy continue to receive trastuzumab but in combination with another chemotherapy, an approach not yet demonstrated effective in randomized trials (Hortobogyi 2005; Montemurro et al 2006). With trastuzumab now incorporated into the adjuvant setting, it is increasingly important to identify effective management strategies for patients who fail to respond to or relapse after receiving it.

Lapatinib (Tykerb ${ }^{\circledR} /$ Tyverb $^{\circledR}$, GlaxoSmithKline, Research Triangle Park, NC, USA), a small molecule inhibitor of tyrosine kinase domains of epidermal growth factor receptor (EGFR or ErbB1) and the ErbB2 (HER2) receptor, inhibits ErbB-driven 
cell growth (Rusnak et al 2001). Lapatinib binds to the ATP-binding site of tyrosine kinase, blocking phosphorylation and activation of the receptor. As a result, activation of extracellular signal-related kinase (ERK)-1/2 and phosphatidylinositol 3' kinase (PI3K)/Akt is blocked (Rusnak et al 2001). In preclinical studies, lapatinib was active against trastuzumab-resistant breast cancer cells (Konecny et al 2006). In a phase I study, lapatinib was active and well tolerated in women with advanced solid tumors when administered in combination with capecitabine at the optimally tolerated dose of lapatinib $1250 \mathrm{mg}$ orally Days 1-21 and capecitabine $2000 \mathrm{mg} / \mathrm{m}^{2}$ Days 1-14 of a 21-day cycle (Schwartz et al 2004). Lapatinib monotherapy has been well tolerated by women with advanced breast cancer in phase II trials (Burstein et al 2004; Blackwell et al 2005; Gomez et al 2006). The response rate was highest in the first-line setting (24\%), but responses to monotherapy were also seen in heavily pretreated patients, including those previously treated with trastuzumab $(4 \%-8 \%)$. Although lapatinib targets two distinct receptor pathways, responses in these trials were limited to patients with ErbB2-positive disease.

\section{Lapatinib plus capecitabine for ErbB2-positive advanced breast cancer}

A phase III randomized, open-label study was conducted comparing lapatinib plus capecitabine with capecitabine monotherapy in women with ErbB2-positive (3+ by immunohistochemistry [IHC] or IHC $2+$ and gene amplification by fluorescence in situ hybridization [FISH]), locally advanced or metastatic breast cancer. Enrollment required that women received prior treatment that included, but was not limited to, an anthracycline, a taxane, and trastuzumab. In the combination therapy arm, lapatinib was administered continuously at a dose of $1250 \mathrm{mg}$ once daily orally and capecitabine $2000 \mathrm{mg} / \mathrm{m}^{2}$ in 2 divided doses on Days 1 through 14 . In the monotherapy arm, capecitabine was administered at a dose of $2500 \mathrm{mg} / \mathrm{m}^{2}$ daily in 2 divided doses on Days 1 through 14. In both arms, treatment was administered on a 21-day cycle. The primary endpoint was time to tumor progression (TTP) (Geyer et al 2006).

Median age was 53 years and $14 \%$ of women were $\geq 65$ years old. Approximately half of the women's tumors were hormone receptor positive and nearly all (96\%) had metastatic disease, with approximately $50 \%$ having metastases to three or more sites. Prior trastuzumab treatment was administered in the metastatic setting in $95 \%$ of patients (Geyer et al 2006; Tykerb 2007).

\section{Planned interim analysis}

Study enrollment was stopped early based on a planned interim analysis of TTP that met criteria for early reporting based on superiority in the capecitabine plus lapatinib arm. At the time of this analysis a total of 324 women had been randomized and there were 49 TTP events in the combination therapy arm and 72 in the monotherapy arm, with a hazard ratio for TTP of 0.49 ( $95 \%$ CI, 0.34 to $0.71 ; p \leq 0.001$ ) based on an evaluation by blinded independent reviewers. The median TTP in the combination arm was 8.4 months and 4.4 months in the monotherapy arm (Geyer et al 2006).

\section{Updated analysis}

By the time enrollment was suspended, an additional 75 women had been enrolled in the study. An updated analysis was conducted on this larger population of 399 women. With a total of 82 TTP events in the combination arm and 102 in the monotherapy arm by independent review, the hazard ratio for TTP remained statistically significant in favor of capecitabine plus lapatinib (HR, 0.57; 95\% CI, 0.43-0.77; $\mathrm{p}=0.00013$ ). This corresponds to an increase in median TTP from 4.3 months in the monotherapy group to 6.2 months in the combination therapy group (Figure 1A). Overall response rate also favored capecitabine plus lapatinib ( $24 \%$ vs $14 \%$ ), and fewer patients treated with the combination developed symptomatic central nervous system progression ( 4 vs 13 patients; $p=0.045$ by Fisher's exact test) (Cameron et al 2007; Tykerb 2007).

Thirty percent of patients had died by the time of the updated analysis. While overall survival trended in favor of capecitabine plus lapatinib, data were not statistically significant (HR, 0.78; 95\% CI, 0.55-1.12. p = 0.177) (Figure 1B) (Cameron et al 2007; Tykerb 2007).

\section{Safety}

The most common adverse events were diarrhea, palmarplantar erythrodysesthesia (PPE), nausea, vomiting, and rash distinct from PPE (Table 1). The incidence of these adverse events was similar in each group, with the exception of diarrhea and rash, both more common in women treated with capecitabine plus lapatinib. A similar percentage of women in each group (14\% in the combination group and 14\% with capecitabine alone) discontinued treatment due to adverse events (Tykerb 2007).

Based on experience with trastuzumab, cardiac events were closely monitored. A cardiac event was defined as any decline in left ventricular ejection fraction (LVEF) that was symptomatic (ie, National Cancer Institute Common Terminology Criteria for Adverse Events [NCI CTCAE] $\geq$ Grade 3) 

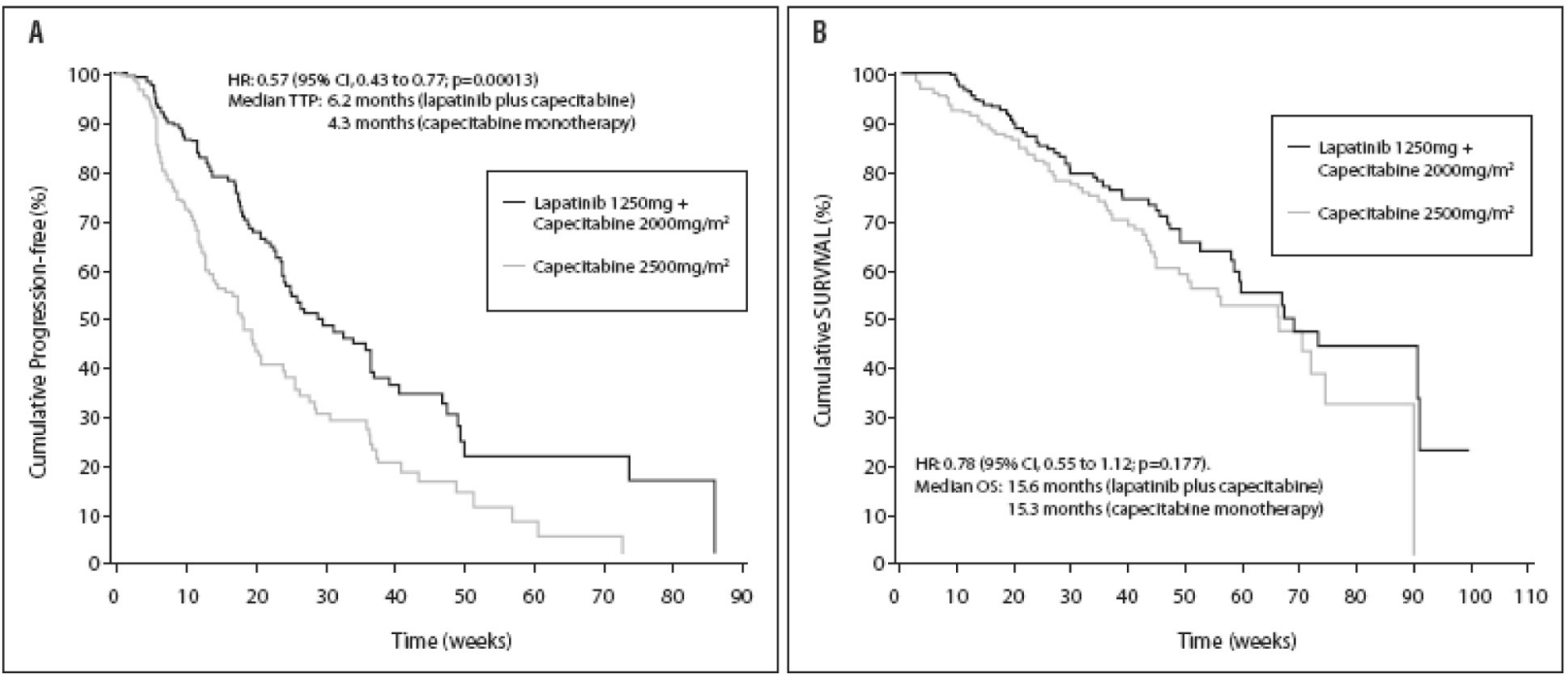

Figure I Kaplan-Meier estimates of time to progression (HR 0.57; $95 \% \mathrm{Cl} 0.43$ to 0.77$) \mathrm{P}=0.000 \mathrm{I} 3$ ) (A) and overall survival (B) in ITT population and based on independent review. Reproduced Cameron D, Martin A-M, Newstat B, et al. 2007. Lapatinib (L) plus capecitabine (C) in HER2+ advanced breast cancer (ABC): updated efficacy and biomarker analysis.J Clin Oncol, 2007 ASCO Annual Meeting Proceedings Part I, 25: 1035.

or an asymptomatic decline that was $\geq 20 \%$ relative to baseline and below the institution's lower limit of normal. There were no differences in the mean LVEF between the two treatment groups (Geyer et al 2006). Four asymptomatic cardiac events of LVEF occurred in patients treated with the combination and two in the monotherapy group. One patient in the combination group experienced Prinzmetal's angina, which resolved when treatment was stopped. This patient experienced a decrease in LVEF 28 days after stopping treatment with lapatinib. On follow-up, LVEF was at or above the lower limit of normal in all patients experiencing cardiac events (Geyer et al 2006; Tykerb 2007).

\section{Initial biomarker data}

Preliminary results from a biomarker companion study to the lapatinib combination trial have recently been reported
(Cameron et al 2007). The objectives of this study were to evaluate correlations between biomarker expression and clinical endpoints (TTP/progression-free survival [PFS]) and to characterize the biomarker predictors of benefit from lapatinib. Levels of EGFR/HER2 were analyzed using both IHC/FISH and ECD assays. Baseline EGFR/ECD levels did not affect PFS in either the combination $(p=0.88)$ or monotherapy arms $(\mathrm{p}=0.32)$. Higher HER2 ECD levels, however, were associated with shorter PFS in the capecitabine monotherapy group $(\mathrm{p}=0.026)$. This was not demonstrated in the lapatinib arm $(\mathrm{p}=0.14)$. When PFS was evaluated with respect to baseline HER2 by ECD, the addition of lapatinib had a positive effect. This effect was seen regardless of ECD level, with a hazard ratio for patients with baseline ECD $\geq 78$ of 0.271 ( $p=0.0013 ; 95 \%$ CI $0.122,0.621)$ and with baseline ECD $\leq 78$ of 0.483 ( $p=0.0018 ; 95 \%$ CI $0.306,0.763)$.

Table I Most frequent adverse events based on National Cancer Institute Common Terminology Criteria for Adverse Events, version 3 (Tykerb 2007)

\begin{tabular}{|c|c|c|c|c|c|c|}
\hline \multirow[t]{2}{*}{ Adverse event } & \multicolumn{3}{|c|}{$\begin{array}{l}\text { Capecitabine + lapatinib } \\
(n=198)\end{array}$} & \multicolumn{3}{|l|}{$\begin{array}{l}\text { Capecitabine }^{b} \\
(n=\mid 9 I)\end{array}$} \\
\hline & All grades, $\%$ & Grade 3, \% & Grade 4, \% & All grades, $\%$ & Grade 3, \% & Grade $4, \%$ \\
\hline Diarrhea & 65 & 13 & 1 & 40 & 10 & 0 \\
\hline PPE & 53 & 12 & 0 & 51 & 14 & 0 \\
\hline Rash & 28 & 2 & 0 & 14 & 1 & 0 \\
\hline Nausea & 44 & 2 & 0 & 43 & 2 & 0 \\
\hline Vomiting & 26 & 2 & 0 & 21 & 2 & 0 \\
\hline Mucosal inflammation & 15 & 0 & 0 & 12 & 2 & 0 \\
\hline
\end{tabular}

aLapatinib $1250 \mathrm{mg}$ once daily orally (continuously) and capecitabine $2000 \mathrm{mg} / \mathrm{m}^{2}$ in two divided doses (Days I through I4) of a 21 -day cycle.

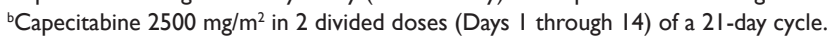

Abbreviations: PPE, palmar plantar erythrodysesthesia. 
The biomarker studies are ongoing and include evaluations of the entire HER2 family, fluoropyrimidine pathway, and identification of potential markers of resistance.

\section{Discussion}

Although metastatic breast cancer (MBC) is incurable, new treatment strategies now allow it to be managed as a chronic disease. Women with MBC are typically treated with sequential regimens. When the first is no longer effective, a second regimen is tried, and then a third (National Comprehensive Cancer Network 2007). This approach might result in extended survival. To date, trastuzumab has been considered the single most effective treatment for women with ErbB2-overexpressing breast cancer. However, controversy exists regarding the optimal duration of treatment and the utility of re-treatment after progression. Results from the recently closed S0347 trial conducted by the Southwest Oncology Group, as well as other on-going phase III trials, will provide important information about the appropriateness of trastuzumab continuation with alternate chemotherapy upon disease progression. Regardless of the outcome of these studies, however, there is a need for alternative treatment options.

Lapatinib plus capecitabine offers a new option for the treatment of women with ErbB2-positive MBC whose disease has progressed following trastuzumab. The addition of lapatinib to capecitabine significantly prolongs TTP, is associated with a trend toward improved survival, and offers the convenience of oral administration. Consistent with earlier results suggesting that lapatinib crosses the blood-brain barrier, (Lin et al 2007), the incidence of symptomatic brain metastases as a first site of progression was significantly reduced when lapatinib was added to capecitabine therapy. Because ErbB2-overexpressing breast cancer appears to be associated with an increased risk of developing brain metastases (Altaha et al 2004) and because trastuzumab

Table 2 On-going randomized phase III trials of lapatinib in patients with breast cancer

\begin{tabular}{|c|c|c|}
\hline Study & Patient population & Randomization \\
\hline EGFI04900 & Trastuzumab-refractory MBC & $\begin{array}{l}\text { Lapatinib + trastuzumab } \\
\text { Lapatinib monotherapy }\end{array}$ \\
\hline EGF3000I & $\begin{array}{l}\text { Ist-line treatment of ErbB2-negative or untested } \\
\text { MBC }\end{array}$ & $\begin{array}{l}\text { Lapatinib + paclitaxel } \\
\text { Paclitaxel + placebo }\end{array}$ \\
\hline EGFI04535 & Ist-line treatment of ErbB2-overexpressing MBC & $\begin{array}{l}\text { Lapatinib + paclitaxel } \\
\text { Paclitaxel + placebo }\end{array}$ \\
\hline EGFI04383 & Ist-line treatment of ErbB2-overexpressing MBC & $\begin{array}{l}\text { Lapatinib + paclitaxel + trastuzumab } \\
\text { Placebo + paclitaxel + trastuzumab }\end{array}$ \\
\hline EGFI08919 & Ist-line treatment of ErbB2-overexpressing MBC & $\begin{array}{l}\text { Lapatinib }+ \text { taxane } \\
\text { Trastuzumab }+ \text { taxane }\end{array}$ \\
\hline EGF30008 & $\begin{array}{l}\text { Ist-line treatment of } \mathrm{HR}+\text { advanced breast } \\
\text { cancer (postmenopausal patients only) }\end{array}$ & $\begin{array}{l}\text { Lapatinib + letrozole } \\
\text { Letrozole + placebo }\end{array}$ \\
\hline CALGB 40302 & $\begin{array}{l}\text { Ist-line treatment of } \mathrm{HR}+\text { advanced breast } \\
\text { cancer (postmenopausal patients only) }\end{array}$ & $\begin{array}{l}\text { Lapatinib + fulvestrant } \\
\text { Placebo + fulvestrant }\end{array}$ \\
\hline EGFI05484 (TEACH) & $\begin{array}{l}\text { Trastuzumab-naïve patients with ErbB2- } \\
\text { overexpressing ESBC who have completed } \\
\text { adjuvant chemotherapy }\end{array}$ & $\begin{array}{l}\text { Lapatinib } \\
\text { Placebo }\end{array}$ \\
\hline EGFI05485 (ALTTO) & $\begin{array}{l}\text { Adjuvant treatment of ErbB2-overexpressing } \\
\text { ESBC }\end{array}$ & $\begin{array}{l}\text { Lapatinib } \\
\text { Trastuzumab } \\
\text { Concurrent trastuzumab/lapatinib } \\
\text { Sequential trastuzumab/lapatinib }\end{array}$ \\
\hline EGFI06903 (Neo-ALTTO) & $\begin{array}{l}\text { Neoadjuvant treatment of ErbB2-overexpressing } \\
\text { ESBC }\end{array}$ & $\begin{array}{l}\text { Lapatinib } \\
\text { Trastuzumab } \\
\text { Concurrent trastuzumab/lapatinib }\end{array}$ \\
\hline NSABP B-4I & $\begin{array}{l}\text { Neoadjuvant treatment of ErbB2-overexpressing } \\
\text { ESBC }\end{array}$ & $\begin{array}{l}\text { Lapatinib } \\
\text { Trastuzumab } \\
\text { Concurrent trastuzumab/lapatinib }\end{array}$ \\
\hline
\end{tabular}

Abbreviations: ALTTO, Adjuvant Lapatinib and/or Trastuzumab Treatment Optimization; CALGB, Cancer and Leukemia Group B; ESBC, early stage breast cancer; DFS, disease-free survival; $\mathrm{HR}+$, hormone-receptor positive; MBC, metastatic breast cancer; NSABP, National Surgical Adjuvant Breast and Bowel Project; TEACH,Tykerb Evaluation after Chemotherapy. 
does not appear to cross the blood-brain barrier (Clayton et al 2004; Stemmler et al 2006) this clinically significant finding deserves further study.

Trastuzumab is associated with an increased risk of cardiotoxicity, particularly when co-administered with anthracyclines (Seidman et al 2002). Many women with advanced breast cancer will have received anthracyclines and trastuzumab. While lapatinib is not free of cardiac effects, the risk appears to be low. In an analysis of cardiac safety in 3127 patients treated with lapatinib in clinical trials, the incidence of lapatinib-associated asymptomatic LVEF decreases was $1.3 \%$. Symptomatic decreases were rare and generally reversible (Perez et al 2006).

The combination of lapatinib and capecitabine fills a void in the treatment of women with ErbB2-overexpressing advanced breast cancer who have received prior therapy with an anthracycline, a taxane, and trastuzumab. Ongoing research is investigating the use of lapatinib alone, in combination with various cytoxic and biologic agents, in combination with trastuzumab, and in adjuvant therapy for early breast cancer (Table 2).

\section{References}

Altaha R, Crowell E, Ducatman B, et al. 2004. Risk of brain metastases in HER2/neu-positive breast cancer. J Clin Oncol, 2004 ASCO Annual Meeting Proceedings (Post-Meeting Edition), 22:682.

American Cancer Society. 2007. Cancer Facts and Figures 2006 [online]. Accessed 21 June 2007. URL: http:// www.cancer.org/docroots/STT/ content/STT_1x_Cancer_Facts_Figures_2006.asp.

Blackwell KL, Burstein H, Pegram M, et al. 2005. Determining relevant biomarkers from tissue and serum that may predict response to single agent lapatinib in trastuzumab refractory metastatic breast cancer. J Clin Oncol, 23:193s. Abstract 3004. [Updated based on presentation.]

Burstein H, Storniolo AM, Franco S, et al. 2004. A phase II, open-label, multicenter study of lapatinib in two cohorts of patients with advanced or metastatic breast cancer who have progressed while receiving trastuzumab-containing regimens. Ann Oncol, 15(suppl 3):27(abstract 104O).

Cameron D, Martin A-M, Newstat B, et al. 2007. Lapatinib (L) plus capecitabine $(\mathrm{C})$ in HER2+ advanced breast cancer (ABC): updated efficacy and biomarker analysis. J Clin Oncol, 2007 ASCO Annual Meeting Proceedings Part I, 25:1035.

Clayton AJ, Danson S, Jolly S, et al. 2004. Incidence of cerebral metastases in patients treated with trastuzumab for metastatic breast cancer. $\mathrm{Br} J$ Cancer, 91:639-43.
ESMO Guidelines Working Group. 2007. Recurrent or metastatic breast cancer: ESMO Clinical Recommendations for diagnosis, treatment and follow-up. Ann Oncol, 18(Suppl 2):ii9-ii11.

Geyer CE, Forster J, Lindquist D, et al. 2006. Lapatinib plus capecitabine for HER2-positive advanced breast cancer. $N$ Engl J Med, 355:2733-43.

Gomez HL, Chavez MA, Doval DC, et al. Results from a phase II randomized study of lapatinib as first-line treatment for patients with HER2amplified locally advanced or metastatic breast cancer. Poster presentation at the 29th Annual San Antonio Breast Cancer Symposium. 2006; Abstract 1090. available at: http://www.posters2view.com/sabcs06/view. php?nu=1090 Accessed June 19, 2007.

Heimann R, Hellman S. 2000. Clinical progression of breast cancer malignant behavior: what to expect and when to expect it. J Clin Oncol, 18:591-9.

Hortobagyi GN. 2005. Continuation of trastuzumab beyond disease progression (letter to the editor). J Clin Oncol, 23:2868-9.

Konecny GE, Pegram MD, Venkatesan N, et al. 2006. Activity of the dual kinase inhibitor lapatinib (GW572016) against HER-2-overexpressing and trastuzumab-treated breast cancer cells. Cancer Res, 66:1630-9.

Lin NU, Dieras V, Paul, D, et al. 2007 Phase II trial of lapatinib for brain metastases in patients with HER2+ breast cancer. J Clin Oncol, ASCO Annual Meeting Proceedings Part I, 25:1012.

Montemurro F, Donadio M, Clavarezza M, et al. 2006. Outcome of patients with HER2-positive advanced breast cancer progressing during trastuzumab-based therapy. The Oncologist, 11:318-24.

National Comprehensive Cancer Network. 2007. NCCN clinical practice guidelines in oncology: Breast Cancer v.2.2007 [online]. Accessed 21 June 2007. URL: http://www.nccn.org.

Perez EA, Byrne JA, Hammond IW, et al. 2006. Cardiac safety experience in 3127 patients (pts) treated with lapatinib. Ann Oncol, 17(suppl 8):ix70.

Rusnak DW, Lackey K, Affleck K, et al. 2001. The effects of the novel, reversible epidermal growth factor receptor/ERbB-2 tyrosine kinase inhibitor, GW2016, on the growth of human normal and tumor-derived cell lines in vitro and in vivo. Mol Cancer Therap, 1:85-94.

Schwartz G, Chu QS-C, Hammond LA, et al. 2004. Phase I clinical, biology and pharmacokinetic study of the combination of GW 572016 and capecitabine in patients with advanced solid tumors (abstract 3070). $J$ Clin Oncol, 22(14S).

Seidman AD, Hudis C, Pierri MK, et al. 2002. Cardiac dysfunction in the trastuzumab clinical trials experience. J Clin Oncol, 20:1215-21.

Slamon DJ, Clark GM, Wong SG, et al. 1987. Human breast cancer: correlation of relapse and survival with amplification of the HER-2/ neu oncogene. Science, 235:177-82.

Stemmler J, Schmitt M, Willems A, et al. 2006. Brain metastases in HER2overexpressing metastatic breast cancer: comparative analysis of trastuzumab levels in serum and cerebrospinal fluid (abstract). J Clin Oncol, 2006 ASCO Annual Meeting Proceedings (Post-Meeting Edition), 24:1525.

Tykerb (lapatinib) tablets. 2007. Product information. Research Triangle Park, NC: GlaxoSmithKline. 
